\title{
Los valores del cuerpo en los jóvenes. ¿Sin valores o nueva valorización? Body values in teenagers. No values or new valorization?
}

*Lorena Velasco-Santos, **José Luis Pastor Pradillo, ***David Blanco-Alcántara, ***Alfredo Jiménez Eguizábal *Junta de Castilla y León (España), **Universidad de Alcalá (España), ***Universidad de Burgos (España)

\begin{abstract}
Resumen. La literatura científica viene insistiendo en los últimos años en una nueva valorización del cuerpo que el presente artículo trata de estudiar empíricamente. El instrumento de medida es el test elaborado por Casares y Collados (1998) que clasifica en 10 los valores del cuerpo y se ha aplicado a una muestra de 536 estudiantes de $1^{\circ}$ de Bachillerato en Castilla y León en el curso escolar 2017/18. Se llevan a cabo dos procedimientos diferenciados en la obtención de resultados, por palabras y por valores del cuerpo. Asimismo, en cada uno de estos procedimientos se analiza tanto las frecuencias máximas como los valores medios, derivando finalmente en tres vías diferentes. Las puntuaciones obtenidas según las tres metodologías presentan características distintas y no susceptibles de comparativa dada la heterogeneidad en las unidades de medida, mientras sus jerarquías derivadas sí permiten comparación al respecto. Se estudian las tendencias más generalizadas que vinculan a los estudiantes con la sociedad occidental contemporánea y el cuerpo postmoderno. Los resultados muestran el valor placer como el más agradable y el religioso como el menos. Asimismo, los valores del cuerpo biológico, estético, intelectual y afectivo-social son destacados como los más agradables después del placer, mientras los valores ecológico, ético y dinámico son destacados como menos agradables. Todo ello apoya en general la perspectiva descrita por la literatura sobre el cuerpo postmoderno y su gran valoración como estructura orgánica en detrimento de su función humana, repercutiendo en campos diversos que demandan una intervención.
\end{abstract}

Palabras clave. Educación Física, Valores, Cuerpo, Currículum, Jerarquía axiológica, Estudiantes de Instituto.

Abstract. The scientific literature has been insisting in recent years on a new valorization of the body that this article tries to study empirically. The instrument of measurement is the test devised by Casares and Collados (1998), which divides body values into 10 categories and it has been applied to a sample of 536 students from 1st year of Bachillerato in Castilla y León region of Spain during the academic year 2017/18. Two different procedures are performed to obtain results, by words and by body values. Also, in each of these procedures, both the maximum frequencies and the mean values are analyzed, eventually resulting in three different routes. The results obtained from the three methodologies show different characteristics and are not susceptible of comparative due to the heterogeneity in the units of measurement, however, the hierarchies derived from them do allow comparison in this regard. The most common trends linking students with contemporary Western society and post-modern body are examined. The results show pleasure value as the most fulfilling and religion as the least. Likewise, the biological, aesthetic, intellectual and affective-social body values are highlighted as the most pleasing after pleasure value, while the ecological, ethical and dynamic values are highlighted as less pleasant. All this, generally speaking, supports the perspective described by the literature about the postmodern body and its great value as an organic structure to the detriment of its human function, impacting on various fields that require intervention.

Keywords. Physical Education, Values, Body, Curriculum, Axiological Hierarchy, Secondary School Students.

\section{Introducción}

El valor y la significatividad del cuerpo están marcados hoy, tal vez más que en ninguna otra época anterior, por la fragmentariedad. La nueva valorización del cuerpo está motivando una atención investigadora específica en torno al sentido y magnitud de los cambios operados, así como sobre sus repercusiones culturales y educativas, en unas circunstancias como las actuales. Compartiendo la visión melancólica dibujada por Baudrillard (2002 y 2009), se advierte en las sociedades de postmodernidad un cuerpo humano al servicio de la apariencia y la productividad. La nueva sociedad prima el rendimiento en cantidad sobre la esencia de calidad, la satisfacción y los objetivos fáciles de conseguir e inmediatos frente al sacrificio y el ejercicio de voluntad que exige o demanda el largo plazo.

Diferentes autores han intentado configurar el mapa de aproximación a la concepción social del cuerpo en la cultura actual, demostrando la existencia de un sesgo material, con importantes repercusiones educativas. Así, Gervilla (2000) resume el cuerpo postmoderno como cuerpo: sex-ducción, narcisista, estético e indoloro o del post-deber. Del mismo

Fecha recepción: 17-01-20. Fecha de aceptación: 01-08-20

Lorena Velasco Santos

lorenavelascoes@yahoo.es modo, Lipovetsky (2000) establece cuatro ámbitos: sexo, narcisismo, estética e indoloro. García y Alemany (1996, p.106) resumen las tendencias actuales en: atención y cuidado hedonista, cuerpo admirable y cuerpo como valor instrumental. Siguiendo a Ribeiro (2003), se establecen cuatro categorías: bienestar, energía y destreza, placer y apariencia. Asimismo, Vázquez (2001) señala tres indicadores principales del culto al cuerpo: la belleza, la salud y el rendimiento. Pastor (2009, p.11) subraya como principales características del cuerpo postmoderno: saludable, aparente y hedonista. Por su parte, García y Alemany (1996, p.105) explican que el hombre de hoy le presta más atención al cuerpo que el de épocas inmediatamente anteriores, y lo utiliza sólo en una determinada línea, exhibicionista o hedonista. Siguiendo a Le Breton (2002, p.28), la concepción moderna del cuerpo implica «que el hombre se ha separado del cosmos (ya no es el macrocosmos el que explica la carne, sino una anatomía y una fisiología que sólo existen en el cuerpo), de los otros (pasaje de una sociedad de tipo comunitaria a una sociedad de tipo individualista en la que el cuerpo es la frontera de la persona) y, finalmente, de sí mismo (el cuerpo está planteado como algo diferente de él)».

En el contexto de esta cultura del cuerpo postmoderno, muy alejada de preocupaciones esencialistas, son muchos los autores que demandan una verdadera corporeidad en la 
educación y la escuela, y «un sentido educativo de lo corporal» Águila y López (2019, p. 413) ya que «nuestra consciencia de la realidad se basa en nuestras experiencias directas y todas ellas pasan por nuestro cuerpo» (p. 414).

Emerge así la necesidad de aproximarse al núcleo mismo de los valores del cuerpo contrastando la realidad descrita por la literatura científica con los resultados empíricos obtenidos de los valores del cuerpo de jóvenes. Determinar la envergadura del giro en la valorización del cuerpo y conocer la intensidad de sus principales dimensiones medulares contribuirán en la dimensión pedagógica a la discusión teórica y a una revisión de gran calado de los valores, contenidos y métodos utilizados en los procesos de escolarización para construir la identidad personal y los modos de sociabilidad.

Antes de poder realizar cualquier intervención en el aula, con el propósito de actuar sobre los valores, se debe conocer la realidad que acontece. Así, la presente investigación tiene por objetivo analizar y estudiar los valores del cuerpo de los estudiantes de primero de Bachillerato en Castilla y León en el curso 2017-2018, contrastando su coincidencia, o no, con los valores teorizados en la literatura actual según la cual los estudiantes se verían inmersos en la corriente social hegemónica que legitima un cuerpo deshumanizado, reproduciendo valores del cuerpo que emergen de su realidad más material, haciendo a su vez pervivir este grabado que aseguraría la permanencia del presente modelo.

\section{Metodología}

A continuación se exponen los aspectos más relevantes en cuanto al diseño y metodología de la investigación. Se aborda la muestra objeto del estudio, el instrumento de medida utilizado, así como la metodología o procedimiento llevado a cabo.

Cabe señalar que todos y cada uno de estos aspectos se tornan de especial relevancia en la investigación, puesto que cada uno de ellos puede condicionar los resultados obtenidos. La selección de la muestra y la elección del instrumento de medida deben ser, y han sido, tratados con especial cautela, asegurando en todo momento las propiedades de representatividad, fiabilidad y validez. Pero se debe poner el foco de atención, como se explica con más detalle en la sección que muestra el procedimiento, en la metodología a emplear puesto que, partiendo de la misma muestra, con el mismo instrumento de medida y, por consiguiente, con los mismos datos, se derivan resultados diferentes en función de la metodología empleada. Es por ello que se antoja de vital importancia identificar y seleccionar la metodología más idónea a las características y objetivo de la investigación.

\section{Participantes}

El estudio se dirige a los alumnos matriculados en $1^{\circ}$ Bachillerato en Castilla y León en el curso 2017-2018. Se solicita participación a los 274 centros con oferta formativa en $1^{\circ}$ Bachillerato en esta comunidad autónoma, siendo 60 los centros finalmente interesados en participar. El estudio se focaliza en los alumnos de $1^{\circ}$ de Bachillerato al tratarse del único curso donde se imparte Educación Física con alumnos mayores de 17 años por recomendaciones específicas del test o instrumento de medida y su complejidad léxica.
La población total de estudiantes es de 15.894 y la muestra escogida es de 536 estudiantes, lo cual supera notablemente los 376 estudiantes demandados como muestra mínima para poblaciones finitas (tamaño inferior a 100.000 elementos) según muestreo aleatorio simple (ver ecuación 1).

$$
\mathrm{n}=\frac{\mathrm{Z}^{2} \times \mathrm{N} \times \mathrm{p} \times \mathrm{q}}{\mathrm{E}^{2} \times(\mathrm{N}-1)+\mathrm{Z}^{2} \times \mathrm{p} \times \mathrm{q}}=\frac{1.96^{2} \times 15894 \times 0.5 \times 0.5}{0.05^{2} \times(15894-1)+1.96^{2} \times 0.5 \times 0.5}=375,12
$$

donde:

$\mathrm{n}=$ tamaño de la muestra que se quiere calcular.

$\mathrm{Z}=$ desviación del valor medio que se acepta para lograr el nivel de confianza deseado.

$\mathrm{N}=$ tamaño de la población o universo.

$\mathrm{p}=$ proporción de la población que posee la característica objeto de estudio.

$\mathrm{q}=1-\mathrm{p}$.

$\mathrm{E}=$ el error máximo admitido.

Así, el nivel de confianza se establece en un 95\%, valor que conduce a un parámetro $Z=1,96$. El tamaño de la población $(\mathrm{N})$ asciende a 15.894 estudiantes. La proporción de la población que posee la característica objeto de estudio no es conocida, por lo que se aplica el valor que exige un mayor tamaño de muestra para garantizar el proceso, así el parámetro q es 0.5 , resultado de la ecuación (1-p). Y, finalmente, el error máximo admitido es del 5\%.

La muestra escogida queda distribuida de manera proporcional a los porcentajes mantenidos por la población en las variables sexo, modalidad de bachillerato, naturaleza, titularidad y provincia de pertenencia del centro de estudios, según los datos facilitados por la Consejería de Educación de la Junta de Castilla y León. Al mismo tiempo, la muestra de participantes por centro trata de ser lo más equitativa sin perjuicio de la proporción establecida para cada una de las variables contempladas. Así, la muestra seleccionada queda distribuida como se recoge en la tabla 1.

\begin{tabular}{llccc}
$\begin{array}{l}\text { Tabla } 1 . \\
\text { Población y muestra según las } 5 \text { variables de perfil consideradas. }\end{array}$ & & \\
\hline \multirow{2}{*}{ Sexo } & Variables & Población & Muestra & $\begin{array}{c}\% \\
\text { Población }\end{array}$ \\
\hline \multirow{2}{*}{ Modalidad } & Hombres & 7.598 & 254 & $3,34 \%$ \\
& Mujeres & 8.296 & 382 & $3,40 \%$ \\
\hline \multirow{2}{*}{ Ubicación } & Ciencias & 7.714 & 259 & $3,36 \%$ \\
& HH y CC SS & 7.390 & 251 & $3,40 \%$ \\
& Artes & 786 & 26 & $3,31 \%$ \\
\hline \multirow{2}{*}{ Naturaleza } & Urbano & 11.205 & 367 & $3,28 \%$ \\
& Rural & 4.689 & 169 & $3,60 \%$ \\
\hline \multirow{5}{*}{ Provincia } & Público & 12.307 & 412 & $3,35 \%$ \\
& Privado-concertado & 3.587 & 124 & $3,46 \%$ \\
\hline & Ávila & 1.060 & 32 & $3,02 \%$ \\
& Burgos & 2.331 & 119 & $5,11 \%$ \\
& León & 3.068 & 86 & $2,80 \%$ \\
& Palencia & 973 & 33 & $3,39 \%$ \\
& Salamanca & 2.308 & 81 & $3,51 \%$ \\
& Segovia & 1.040 & 34 & $3,27 \%$ \\
Total & Soria & 612 & 24 & $3,92 \%$ \\
\hline Fula & Valladolid & 3.377 & 94 & $2,78 \%$ \\
\hline Zamora & Total & 1.125 & 33 & $2,93 \%$ \\
\hline
\end{tabular}

Fuente: Elaboración propia.

\section{Instrumento}

El instrumento de medida empleado en la presente investigación ha sido el test de valores del cuerpo elaborado por Casares y Collados (1998). Este test pretende analizar los valores otorgados al cuerpo por el individuo, para lo cual se proponen 10 categorías, sentidos o valores del cuerpo diferenciados: 1. Biológico, 2. Ecológico, 3. Instrumental, 4. Dinámico, 5. Afectivo-social, 6. Ético, 7. Estético, 8. Religioso, 9. Intelectual y, por último, 10. Placer.

Cabe señalar que este instrumento de medida es una 
forma indirecta de poder obtener una puntuación cuantitativa de los valores del cuerpo. El valor que un individuo pueda dar a su cuerpo es una variable no observable, por lo que se hace necesario recurrir a un mecanismo que ayude y contribuya a obtener una medida mesurable de la misma.

El fundamento de este test reside en la reacción valorativa de agrado, desagrado o indiferencia experimentada al leer o escuchar algunas palabras, donde el individuo debe indicar el tipo e intensidad de su reacción. En total consta de 250 palabras-expresiones agrupadas en bloques de 25 palabras, donde cada bloque se corresponde con una de las 10 categorías o sentidos de valor del cuerpo previamente mencionados.

Siguiendo a Gervilla $\left(2000^{\mathrm{a}}\right.$, p.219-220), se detalla la connotación asumida para cada uno de los 10 valores del cuerpo, anunciando entre paréntesis los 25 ítems que el autor dedica a cada una de las 10 categorías de cuerpo:

1. Biológico (1-25): abarca predominantemente cuantos valores se refieren al cuerpo como materia viva, así como los relativos a su conservación, desarrollo y cuidado material.

2. Ecológico (26-50): comprende prioritariamente los valores del cuerpo como parte de la naturaleza; como receptor de los bienes de su entorno natural, así como los referentes a su relación con ella: custodia, transformación, disfrute, etc.

3. Instrumental (51-75): se refiere, de modo prioritario, a los valores del cuerpo entendido como medio para realizar tareas diversas, así como para construir, modificar o utilizar todo tipo de materiales u objetos con finalidad práctica y eficaz.

4. Dinámico (76-100): se consideran valores dinámicos los relativos, prioritariamente, al cuerpo en movimiento y a sus posibilidades materializadas en actos perceptibles, con un sentido lúdico, deportivo, de ocio...

5. Afectivo-social (101-125): comprende prioritariamente los valores del cuerpo como expresión de sentimientos, estados de ánimo y/o formas de comunicación con los demás.

6. Ético (126-150): comprende, sobre todo, aquellos valores del cuerpo relacionados con la bondad o malicia de los actos humanos, en relación consigo mismo y con los demás.

7. Estético (151-175): abarca prioritariamente los valores relacionados con la percepción, creación y expresión de la belleza y el arte, en el cuerpo y mediante el cuerpo.

8. Religioso (176-200): se consideran valores religiosos los relacionados directamente con actividades y modos de expresión corporales relativos a la fe y las creencias religiosas.

9. Intelectual (201-225): valores relacionados prioritariamente con la base corporal de las funciones cognitivas e intelectuales del sujeto, así como con aquellas actividades perceptibles en las que el cuerpo interviene para adquirir o expresar conocimientos.

10. Placer (226-250): se consideran valores corporales de placer, prioritariamente, todos aquellos relacionados con el cuerpo en cuanto nos proporciona bienestar, satisfacción y gozo.

El test se cumplimenta por parte de los estudiantes según unas directrices. Cada una de las propuestas ha de ser calificada rodeando con un círculo o marcando con una cruz una de las alternativas situadas a su derecha, las cuales se gradúan de más a menos agradable en cinco categorías: MA
$=$ Muy agradable, $\mathrm{A}=$ Agradable, $\mathrm{I}=$ Indiferente, $\mathrm{D}=$ Desagradable, y MD = Muy desagradable. Se marca aquella opción que se considere más representativa con la impresión o el interés que le suscite, teniendo en cuenta que los ítems marcados con un asterisco deben ser calificados al revés, es decir, cuando algo fuera agradable, habría de marcarse desagradable. No deben dejarse contestaciones sin responder. No es necesario detenerse mucho tiempo para pensar la puntuación a cada término, sino que por el contrario se trata de dar una respuesta lo más intuitiva posible puesto que es esta primera idea o intuición la que se demuestra como más sincera y fiel a la realidad de valor. Es importante ajustar la intensidad de la reacción que produzca cada palabra a la escala de respuestas. No hay tiempo límite.

La corrección del test se ha llevado a cabo según las indicaciones descritas por sus autores (Casares y Collados, 1998). Se han separado las categorías de cuerpo en las diez dispuestas asignando a cada una de ellas los veinticinco ítems que se relacionan, y multiplicando cada una de las respuestas por un coeficiente en función de la calificación marcada. Las respuestas muy agradables se multiplican por +2 , las agradables por +1 , las indiferentes por 0 , las desagradables por -1 y las desagradables por -2 . Una vez realizado este paso, se procede a la suma de las veinticinco puntuaciones obtenidas que conforman cada categoría de valor del cuerpo, siendo esta suma la puntuación final otorgada a ese valor del cuerpo del individuo. Así, esta puntuación final, deberá encontrarse en el intervalo comprendido entre la puntuación máxima de +50 y mínima de -50 .

\section{Propiedades psicométricas}

Antes de comenzar con los cálculos propiamente dichos, se lleva a cabo un estudio de fiabilidad para el caso concreto de la muestra objeto de estudio. Se comprueba, mediante el cálculo del alfa de Cronbach, que el instrumento es fiable para la muestra de estudiantes escogida. Este estadístico permite comprobar la consistencia interna para la escala total, concluyendo que dicho instrumento presenta adecuadas propiedades psicométricas. A pesar de ser el alfa de Cronbach un estadístico ampliamente utilizado en la literatura para comprobar la fiabilidad de este tipo de instrumentos de recogida de datos, cabe señalar que no existe acuerdo unánime que determine el valor exacto a partir del cual se pueda afirmar la fiabilidad del instrumento de medida. Si bien, un instrumento de medida puede considerarse fiable si muestra un valor superior a $0.7-0.8$, siendo tanto más idóneo cuanto más se acerque al valor 1. En este caso, el Alfa de Cronbach toma un valor de 0,956 .

\section{Procedimiento}

Seguidamente, se inician los cálculos para la obtención de resultados siguiendo dos procedimientos diferenciados: por palabras y por valores del cuerpo.

El primer procedimiento, identificado como el procedimiento por palabras, consiste en realizar en primer lugar los análisis necesarios para cada una de las 250 palabras que conforman el cuestionario global para, posteriormente, obtener los valores del cuerpo mediante la aplicación de las directrices que establece este instrumento de medida, agregando cada uno de los 25 ítems que dan lugar a cada categoría de 
valor del cuerpo.

El segundo procedimiento, que se identifica como el procedimiento por valores del cuerpo, consiste en realizar los análisis necesarios para cada categoría de valor del cuerpo, los cuales se obtienen en un primer paso mediante el tratamiento de las respuestas a cada uno de los 250 ítems, agrupados en 10 grupos de 25 , y definiendo en cada caso un valor del cuerpo de acuerdo con las directrices prescritas en el instrumento de medida.

Al mismo tiempo, cada uno de estos dos procedimientos puede ser ejecutado atendiendo a dos análisis diferenciadas: de las máximas frecuencias o de los valores medios.

Cada una de estas formas de realizar el estudio, por palabras o por valores, conduce a la obtención de una clasificación de los valores del cuerpo de los encuestados y su consecuente jerarquización. De esta forma, en la tabla 2, de doble entrada, se muestran las alternativas que se presentan a la hora de abordar el tratamiento de los datos y el consiguiente estudio de los mismos, obteniendo para cada una de ellas una jerarquización de valores.

Como puede observarse, la jerarquía resultante del análisis de valores medios de palabras y la de valores medios de las categorías de valores del cuerpo son coincidentes, por lo que finalmente se exponen tres metodologías diferentes en el tratamiento y análisis de los datos de origen, y consecuentemente tres jerarquías derivadas.

Tabla 2

\begin{tabular}{clll}
\multicolumn{2}{l}{ Vías de obtención de resultados. } & \multicolumn{2}{c}{ Procedimiento } \\
\cline { 2 - 3 } & & Palabras & Valores del cuerpo \\
\hline VALOR & Máxima frecuencia & Jerarquía 1 & Jerarquía 3 \\
APLICADO & Valor medio & Jerarquía 2 & Jerarquía 2 \\
\hline Fuente: Elaboración propia. & &
\end{tabular}

\section{Análisis estadístico de los datos}

En este estudio se trabaja con opciones avanzadas de la hoja de cálculo Excel, así como con el programa estadístico SPSS v.23. Concretamente se calculan estadísticos descriptivos tales como frecuencias absolutas, relativas, valores máximos, mínimos, medios y medidas de dispersión como la desviación típica. Paralelamente, se aplican procedimientos metodológicos enmarcados en la estadística inferencial como los test de diferencia de medias. A este respecto, se emplean pruebas paramétricas cuando se cumplen las condiciones de aplicación de las mismas (distribución normal valorada mediante la prueba de Kolmogorov-Smirnov) y no paramétricas, concretamente se aplica la prueba U de MannWhitney para las comparaciones entre 2 grupos y la prueba H-de Krusal-Wallis para las comparaciones entre más de 2 grupos.

\section{Resultados}

Descritas las tres vías o metodologías empleadas, se exponen los resultados de todas ellas de manera conjunta para facilitar la comparación de resultados.

Es importante explicar detalladamente los pasos previos a la obtención de resultados según el procedimiento por palabras para lo cual se incluyen 2 tablas. La primera de ellas muestra un fragmento de la tabla que recoge el análisis de las frecuencias de las 250 palabras que conforman el cuestionario global para el conjunto de los 536 estudiantes. Por cues-
Tabla 3.

\begin{tabular}{|c|c|c|c|c|c|c|c|c|c|c|c|}
\hline \multirow{3}{*}{ Acción } & \multicolumn{10}{|c|}{ Frecuencias } & \multirow{3}{*}{ V.M.F } \\
\hline & \multicolumn{5}{|c|}{ Absolutas } & \multicolumn{5}{|c|}{ Relativas (cifras en \%) } & \\
\hline & $\mathrm{Ma}$ & $\mathrm{A}$ & $\mathrm{I}$ & $\mathrm{D}$ & $\mathrm{Md}$ & $\mathrm{Ma}$ & A & I & $\mathrm{D}$ & Md & \\
\hline 1. Abrigarse & 97 & 269 & 131 & 34 & 5 & 18,10 & 50,19 & 24,44 & 6,34 & 0,93 & A \\
\hline 2. Alimentarse & 274 & 226 & 28 & 5 & 3 & 51,12 & 42,16 & 5,22 & 0,93 & 0,56 & Ma \\
\hline 3. Amamantar & 39 & 76 & 337 & 47 & 37 & 7,28 & 14,18 & 62,87 & 8,77 & 6,90 & I \\
\hline 8. Dormir & 430 & 82 & 16 & 6 & 2 & 80,22 & 15,30 & 2,99 & 1,12 & 0,37 & Ma \\
\hline 27. Bañarse en el mar/río & 237 & 210 & 56 & 25 & 8 & 44,22 & 39,18 & 10,45 & 4,66 & 1,49 & Ma \\
\hline 32. Hacer camping & 115 & 240 & 141 & 33 & 7 & 21,46 & 44,78 & 26,31 & 6,16 & 1,31 & \\
\hline 43. Podar & 30 & 92 & 286 & 107 & 21 & 5,60 & & 53,36 & 19,96 & & \\
\hline 44. Recolectar & 52 & 143 & 239 & 79 & 23 & 9,70 & 26,68 & 44,59 & 14,74 & 4,29 & \\
\hline 58. Coger & 42 & 127 & 350 & 15 & 2 & 7,84 & & 65,30 & 2,80 & 0,37 & \\
\hline 59. Conducir & 213 & 210 & 91 & 17 & 5 & 39,74 & 39,18 & 16,98 & 3,17 & 0,93 & Ma \\
\hline 60. Coser & 18 & 93 & 238 & 135 & 52 & 3,36 & & 44,40 & 25,19 & 9,70 & I \\
\hline 67. Limpiar el polvo & 8 & 42 & 194 & 208 & 84 & 1,49 & 7,84 & 36,19 & 38,81 & 15,67 & D \\
\hline 80. Balanc & 104 & 180 & 217 & 28 & 7 & 19,40 & & 40,49 & 5,22 & 1,31 & \\
\hline 81. Balonces & 131 & 181 & 149 & 57 & 18 & 24,44 & 33,77 & 27,80 & 10,63 & 3,36 & A \\
\hline 82. Bucear & 249 & 188 & 75 & 19 & 5 & 46,46 & & 13,99 & 3,54 & 0,93 & \\
\hline 93. Pase & 204 & 256 & 60 & 13 & 3 & 38,06 & 7,76 & 11,19 & 2,43 & 0,56 & A \\
\hline 101. Abr & 381 & 117 & 32 & 1 & 5 & 71,08 & & 5,97 & 0,19 & 0,93 & $\mathrm{Ma}$ \\
\hline 102. Aca & 348 & 155 & 24 & 6 & 3 & 64,93 & & 4,48 & 1,12 & 0,56 & Ma \\
\hline 123. Ton & 88 & 192 & 221 & 28 & 7 & 16,42 & 35,82 & 41,23 & 5,22 & 1,31 & \\
\hline 125. *Llorar & 121 & 146 & 106 & 110 & 53 & 22,57 & 27,24 & 19,78 & 20,52 & 9,89 & \\
\hline 127. Ayud & 223 & 276 & 27 & 9 & 1 & 41,60 & 49 & 5,04 & 1,68 & 0,19 & \\
\hline 128. Castidad & 12 & 39 & 198 & 119 & 168 & 2,24 & 7,28 & 36,94 & 22,20 & 31,34 & I \\
\hline 149. *Prostituirse & 206 & 86 & 158 & 37 & 49 & 38,43 & 16,04 & 29,48 & 6,90 & 9,14 & $\mathrm{Ma}$ \\
\hline 150. *Racis & 341 & 51 & 74 & 16 & 54 & 63,62 & & 13,81 & 2,99 & 10, & Ma \\
\hline 153. Ballet & 69 & 90 & 237 & 73 & 67 & 12,87 &, 79 & 44,22 & 13,62 & 12,50 & \\
\hline 1. "Es & 227 & 200 & 91 & 13 & 5 & 42,35 & & & 2,43 & & \\
\hline 170. Ser & 207 & 245 & 75 & 7 & 2 & 38,62 & 71 & 13,99 & 1,31 & 0,37 & A \\
\hline 174. Ten & 213 & 234 & 78 & 8 & 3 & 39,74 & & 14,55 & 1,49 & 0,5 & \\
\hline 178. Аyu & 15 & 67 & 244 & 97 & 113 & 2,80 & 12,50 & 45,52 & 18,10 & 21,08 & \\
\hline 179. Bau & 8 & 124 & 245 & 64 & 65 & 7,09 & & & 11,94 & 12, & \\
\hline 188. Darse la paz & 57 & 200 & 184 & 31 & 64 & 10,63 & 37,31 & 34,33 & 5,78 & 11,94 & A \\
\hline 199. Rezar & 30 & 96 & 228 & 76 & 106 & 5,60 & & & 14,18 & 19,78 & \\
\hline 201. Ana & 109 & 256 & 153 & 14 & 4 & 20,34 & 47,76 & 28,54 & 2,61 & 0,75 & A \\
\hline 202. Apr & & 226 & 41 & 6 & 1 & 48,88 & & 7,65 & 1,12 & 0,1 & Ma \\
\hline 214. Inteli & 299 & 185 & 51 & 1 & 0 & 55,78 & 51 & 9,51 & 0,19 & 0,00 & Ma \\
\hline 216. Inves & & 232 & 85 & 5 & 4 & & & 15,86 & 0,93 & 0,75 & A \\
\hline 226. Aron & 278 & 185 & 67 & 4 & 2 & 51,87 & & 12,50 & 0,75 & 0,37 & $\mathrm{Ma}$ \\
\hline 227. Beber $\mathrm{r}$ & 194 & 216 & 94 & 25 & 7 & & & 17,54 & 4,66 & 1,31 & \\
\hline 240. Paladear & 167 & 168 & 184 & 13 & 4 & 31,16 & 31,34 & 34,33 & 2,43 & 0,75 & I \\
\hline 250. "Una gozada" & 414 & 76 & 41 & 3 & 2 & 77,24 & 14,18 & 7,65 & 0,56 & 0,37 & $\mathrm{Ma}$ \\
\hline
\end{tabular}

250. "Una gozada"

Fuente: Elaboración propia

Nota: Se muestran las abreviaturas de las posibles respuestas, de forma que MA es muy agradable, A es agradable, I es indiferente, D es desagradable y MD es muy desagradable. La

Tabla 4

Principales estadísticos descriptivos de cada palabra.

\begin{tabular}{|c|c|c|c|c|c|}
\hline Acción & $\mathrm{N}$ & Mínimo & Máximo & Media & $\begin{array}{c}\text { Desviación } \\
\text { estándar }\end{array}$ \\
\hline 1. Abrigarse & 536 & -2 & 2 & 0,78 & 0,85 \\
\hline 2. Alimentarse & 536 & -2 & 2 & 1,42 & 0,69 \\
\hline 3. Amamantar & 536 & -2 & 2 & 0,06 & 0,89 \\
\hline 8. Dormir & 536 & -2 & 2 & 1,74 & 0,60 \\
\hline 27. Bañarse en el mar/río & 536 & -2 & 2 & 1,20 & 0,91 \\
\hline 32. Hacer camping & 536 & -2 & 2 & 0,79 & 0,89 \\
\hline 43. Podar & 536 & -2 & 2 & 0,01 & 0,87 \\
\hline 44. Recolectar & 536 & -2 & 2 & 0,23 & 0,96 \\
\hline 58. Coger & 536 & -2 & 2 & 0,36 & 0,68 \\
\hline 59. Conducir & 536 & -2 & 2 & 1,14 & 0,87 \\
\hline 60. Coser & 536 & -2 & 2 & $-0,21$ & 0,95 \\
\hline 67. Limpiar el polvo & 536 & -2 & 2 & $-0,59$ & 0,90 \\
\hline 80. Balancearse & 536 & -2 & 2 & 0,65 & 0,90 \\
\hline 81. Baloncesto & 536 & -2 & 2 & 0,65 & 1,06 \\
\hline 82. Bucear & 536 & -2 & 2 & 1,23 & 0,88 \\
\hline 93. Pasear & 536 & -2 & 2 & 1,20 & 0,77 \\
\hline 101. Abrazo & 536 & -2 & 2 & 1,62 & 0,69 \\
\hline 102. Acariciar & 536 & -2 & 2 & 1,57 & 0,69 \\
\hline 123. Tono de voz & 536 & -2 & 2 & 0,61 & 0,87 \\
\hline 125. *Llorar & 536 & -2 & 2 & 0,32 & 1,29 \\
\hline 127. Ayudar & 536 & -2 & 2 & 1,33 & 0,67 \\
\hline 128. Castidad & 536 & -2 & 2 & $-0,73$ & 1,05 \\
\hline 149. *Prostituirse & 536 & -2 & 2 & 0,68 & 1,29 \\
\hline 150. *Racismo & 536 & -2 & 2 & 1,14 & 1,34 \\
\hline 153. Ballet & 536 & -2 & 2 & 0,04 & 1,15 \\
\hline 161. "Estar bueno/a" & 536 & -2 & 2 & 1,18 & 0,86 \\
\hline 170. Ser atractivo/a & 536 & -2 & 2 & 1,21 & 0,75 \\
\hline 174. Tener buen tipo & 536 & -2 & 2 & 1,21 & 0,78 \\
\hline 178. Ayudar a misa & 536 & -2 & 2 & $-0,42$ & 1,04 \\
\hline 179. Bautismo & 536 & -2 & 2 & 0,01 & 1,06 \\
\hline 188. Darse la paz & 536 & -2 & 2 & 0,29 & 1,12 \\
\hline 199. Rezar & 536 & -2 & 2 & $-0,25$ & 1,13 \\
\hline 201. Analizar & 536 & -2 & 2 & 0,84 & 0,80 \\
\hline 202. Aprender & 536 & -2 & 2 & 1,38 & 0,69 \\
\hline 214. Inteligencia & 536 & -1 & 2 & 1,46 & 0,67 \\
\hline 216. Investigar & 536 & -2 & 2 & 1,19 & 0,79 \\
\hline 226. Aroma & 536 & -2 & 2 & 1,37 & 0,76 \\
\hline 227. Beber refrescos & 536 & -2 & 2 & 1,05 & 0,92 \\
\hline 240. Paladear & 536 & -2 & 2 & 0,90 & 0,90 \\
\hline 250. "Una gozada" & 536 & -2 & 2 & 1,67 & 0,67 \\
\hline
\end{tabular}

tión de dimensiones se ha preferido mostrar solamente un fragmento de dicha tabla con el ánimo de facilitar una mayor comprensión del procedimiento e interpretación de los resultados. En concreto se han seleccionado cuatro ítems de cada 
grupo de 25 palabras que definen cada valor del cuerpo (ver tabla 3). La tabla 4 muestra los principales estadísticos descriptivos para cada palabra. Igualmente, se expone un fragmento de la tabla completa debido al gran tamaño de la misma. Se ha decidido mostrar las mismas palabras señaladas en la anterior tabla.

Por su parte, también es importante reflejar los principales estadísticos descriptivos calculados en el procedimiento de valores medios, recogidos en la tabla 5 , antes de proceder a la síntesis de resultados arrojados por las 3 vías empleadas.

Una vez detallados los pasos previos en ambos procedimientos, por palabras y por valores del cuerpo, se exponen los resultados correspondientes a las tres metodologías previamente descritas. Recuérdese cómo la jerarquía 1 se correspondía con el procedimiento por palabras y análisis de frecuencias; la jerarquía 2 con el análisis de valores medios, bien mediante el procedimiento por palabras o por valores, pues son coincidentes; y la jerarquía 3 con el procedimiento por valores y análisis de frecuencias.

En primer lugar se muestran los resultados relativos a las puntuaciones obtenidas en cada valor del cuerpo, así como la jerarquización derivada de dichas puntuaciones. Seguidamente se realiza la interpretación de los resultados de manera conjunta, señalando los resultados más relevantes desprendidos del estudio. Finalmente se realiza una interpretación de lo observado en cada valor del cuerpo, relacionándolo con lo existente en la literatura.

Así, la tabla 6 muestra las puntuaciones otorgadas por los estudiantes a los valores del cuerpo.

Las puntuaciones obtenidas de las dos primeras metodologías oscilan entre $-50 \mathrm{y}+50$ por lo que pueden esta-

Tabla 5 .

\begin{tabular}{|c|c|c|c|c|c|c|c|c|c|}
\hline \multirow[b]{2}{*}{ Variables } & \multirow[b]{2}{*}{$\mathrm{N}$} & \multirow[b]{2}{*}{ Mín. } & \multirow[b]{2}{*}{ Máx. } & \multirow[b]{2}{*}{ Media } & \multirow{2}{*}{$\begin{array}{l}\text { Desv. } \\
\text { Estand }\end{array}$} & \multicolumn{2}{|c|}{ Asimetría } & \multicolumn{2}{|c|}{ Curtosis } \\
\hline & & & & & & Estadístico & $\begin{array}{c}\text { Error } \\
\text { estándar }\end{array}$ & Estadístico & $\begin{array}{c}\text { Error } \\
\text { estándar }\end{array}$ \\
\hline 1. Biológico & 536 & -14 & 47 & 25,14 & 8,227 & $\begin{array}{c}-0,58 \\
-0\end{array}$ & 0,11 & 1,54 & 0,21 \\
\hline 2. Ecológico & 536 & -31 & 42 & 11,83 & 11,362 & $-0,01$ & 0,11 & 0,49 & 0,21 \\
\hline 3. Instrumental & 536 & -18 & 39 & 8,50 & 8,772 & 0,39 & 0,11 & 0,70 & 0,21 \\
\hline 4. Dinámico & 536 & -19 & 49 & 20,73 & 11,199 & $-0,24$ & 0,11 & 0,25 & 0,21 \\
\hline 5. Afectivo/social & 536 & -21 & 49 & 24,90 & 10,626 & $-0,76$ & 0,11 & 1,29 & 0,21 \\
\hline 6. Ético & 536 & -11 & 43 & 15,92 & 8,544 & 0,04 & 0,11 & 0,38 & 0,21 \\
\hline 7. Estético & 536 & -43 & 50 & 24,26 & 12,820 & $-0,54$ & 0,11 & 1,23 & 0,21 \\
\hline 8. Religioso & 536 & -50 & 49 & $-6,23$ & 20,357 & $-0,37$ & 0,11 & $-0,13$ & 0,21 \\
\hline 9. Intelectual & 536 & -23 & 50 & 24,49 & 12,318 & $-0,23$ & 0,11 & 0,02 & 0,21 \\
\hline 10. Placer & 536 & -37 & 50 & 33,73 & 11,384 & $-1,45$ & 0,11 & 4,44 & 0,21 \\
\hline
\end{tabular}

10. Placer $\quad 536 \quad-37$
Fuente: Elaboración propia.

Tabla 6.

Puntuaciones según las tres metodologías.

\begin{tabular}{lccc}
\multicolumn{1}{c}{ Puntuaciones según las tres metodologías. } \\
\hline \multicolumn{1}{c}{ Valores } & Jerarquía 1 & Jerarquía 2 & Jerarquía 3 \\
\hline 1. Biológico & 30,00 & $25,14(8,22)$ & $6,72 \%(36)$ \\
2. Ecológico & 10,00 & $11,83(11,36)$ & $0,56 \%(3)$ \\
3. Instrumental & 9,00 & $8,5(8,77)$ & $0,19 \%(1)$ \\
4. Dinámico & 24,00 & $20,73(11,19)$ & $5,41 \%(29)$ \\
5. Afectivo/social & 29,00 & $24,9(10,62)$ & $5,78 \%(31)$ \\
6. Ético & 21,00 & $15,92(8,54)$ & $0,75 \%(4)$ \\
7. Estético & 22,00 & $24,26(12,82)$ & $8,96 \%(48)$ \\
8. Religioso & 1,00 & $-6,23(20,35)$ & $1,31 \%(7)$ \\
9. Intelectual & 30,00 & $24,49(12,31)$ & $16,04 \%(86)$ \\
10. Placer & 47,00 & $33,73(11,38)$ & $54,29 \%(291)$ \\
\hline
\end{tabular}

Fuente: Elaboración propia

Nota: En la jerarquía 2 los valores entre paréntesis muestran la desviación típica. En la jerarquía 3 los valores entre paréntesis muestran la frecuencia absoluta.

Tabla 7.

Posicionamiento jerárquico según las tres metodologías

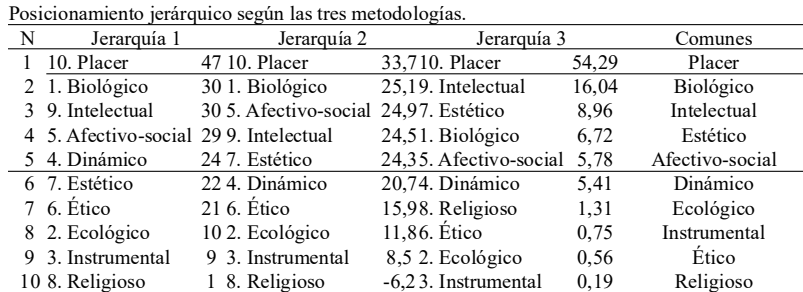
\begin{tabular}{lllll}
108. Religioso & 1 8. Religioso & $-6,23$. Instrumental & 0,19 & Relico \\
\hline & & &
\end{tabular}

Fuente: Elaboración propia. blecerse comparaciones entre ellas. Sin embargo, la tercera metodología queda expresada en forma de porcentajes de tal manera que la suma de todas las categorías equivale a la unidad $(100 \%)$. Esta tercera vía no es equitativa ni comparable con las dos anteriores, si bien, aporta información complementaria muy relevante en el presente estudio.

La tabla 7 representa la jerarquización axiológica derivada de las anteriores puntuaciones.

A diferencia de lo ocurrido con las puntuaciones registradas en las tres metodologías mostradas en la tabla 6, las posiciones descritas por cada valor del cuerpo en cada una de las tres metodologías sí es comparable con el resto puesto que las puntuaciones quedan traducidas en forma de ordenamientos jerárquicos.

A la luz de las tres jerarquías, en términos generales, se pueden distinguir dos tendencias diferenciadas, los cinco valores del cuerpo considerados más agradables y los cinco considerados menos agradables. Si bien, a continuación se detallan las apreciaciones más relevantes de los resultados obtenidos.

\section{Valores placer y religioso}

Puede afirmarse que el valor del cuerpo placer siempre ocupa la primera posición en todas las jerarquías, independientemente de la metodología aplicada. Por su parte, el valor del cuerpo religioso tiende a ocupar la última posición, sin embargo, no presta la rotundidad y homogeneidad presentada por el valor placer y su hegemónica primera posición. El valor religioso ocupa el último puesto en dos de las tres jerarquías mostradas para el total de la muestra de estudiantes. Así, en términos medios, el valor religioso ostenta las valoraciones menos agradables. Sin embargo, al analizar las frecuencias de los valores del cuerpo preferidos por los estudiantes (jerarquía 3), éste no queda posicionado en último lugar. Esta disparidad entre valores medios y preferidos (análisis de frecuencias) puede encontrar su justificación en la alta dispersión mostrada en las respuestas al valor del cuerpo religioso.

Son, en todo caso, el valor placer y el valor religioso los dos valores del cuerpo considerados más estables u homogéneos en todas y cada una de las metodologías empleadas. Estos dos valores definen la primera posición y la última respectivamente en toda jerarquía, con las salvedades previamente descritas para el caso del valor del cuerpo religioso.

Por su parte, como previamente se señalaba, se definen dos grupos de valores, los más agradables y los menos.

\section{Valores biológico, estético, intelectual y afectivo-so- cial}

Existe coincidencia en las cuatro siguientes posiciones al primer puesto, representado por el valor placer, en las jerarquías resultantes de las tres metodologías aplicadas. Los cuatro valores que ocupan así la segunda, tercera, cuarta y quinta posición en las tres jerarquías son el valor biológico, estético, intelectual y afectivo-social. Si bien, el valor estético ostenta el sexto puesto en la jerarquía 1, como única excepción a dicha afirmación.

Valores ecológico, ético, instrumental y dinámico

Los valores dinámico, ético, ecológico e instrumental, 
contrariamente a los cuatro anteriormente señalados como más agradables después del valor placer, coinciden en ocupar en las tres jerarquías del quinto al noveno puesto, es decir, los menos agradables después del valor del cuerpo religioso. Si bien, el valor dinámico ostenta la quinta posición en la jerarquía 1 , como única excepción a dicha afirmación.

\section{Discusión}

Considerando la muestra de estudiantes escogida como reflejo del producto social y su cultura hegemónicamente legitimada, se observa cómo ésta describe patrones descritos por la literatura especializada. Como anuncia Barbero (2007, p.31), «La cultura corporal es el conjunto de valores, apreciaciones, ideas, saberes, usos, prácticas, etc. que expresan lo que puede o debe hacerse con el cuerpo».

Dado que los resultados mostrados en forma de jerarquías presentan coincidencias y generalidades que no pueden ser descritas en los resultados mostrados en forma de puntuaciones, la discusión de resultados es llevada a cabo mediante los resultados obtenidos en forma de posicionamientos jerárquicos en último lugar expuestos.

\section{Valor placer}

El valor del cuerpo placer, que ocupa la primera posición en todas las jerarquías resultantes del estudio empírico, es al mismo tiempo subrayado como hegemónico en la sociedad occidental contemporánea por la gran mayoría de autores previamente detallados. Así, Gervilla (2000) lo denomina valor del cuerpo indoloro o postdeber, Lipovetsky (2000) indoloro, García y Alemany (1996, p.106) atención y cuidado hedonista, y Ribeiro (2003) placer.

La sociedad idolatra y premia el hedonismo, el placer frente al displacer, el disfrute frente al sacrificio, el goce frente al dolor. Del mismo modo, Lipovetsky (2000) señala que desde hace medio siglo se ha puesto en marcha una nueva lógica de secularización de la moral, independiente de toda religión y de todo deber, por cuanto consiste en la disolución del deber mismo. La idea de sacrificio está totalmente deslegitimizada, la moral no exige consagrarse a un fin superior a uno mismo, se estimulan los deseos inmediatos, la pasión del ego, la felicidad intimista y materialista, pasando del Bien al bienestar.

\section{Valor biológico}

El valor del cuerpo biológico es reconocido como uno de los más agradables en el estudio empírico llevado a cabo, y legitimado por la sociedad. El valor biológico del cuerpo reconoce su dimensión más material, su estado de salud y condición físico-motriz. A este respecto, algunos autores destacan dicho valor del cuerpo común en la sociedad bajo la siguiente terminología: Ribeiro (2003) bienestar, energía y destreza, y Vázquez (2001) salud y rendimiento.

En esta línea, Rosseau (1996) recuerda que el valor biológico del cuerpo puede ser un objetivo y herramienta sociopolítica ya que, cuanto mayor debilidad padece el cuerpo, más impera su limitación y ofrece su mandato, y cuanto más vigoroso, más se dispone al servicio del hombre obedeciendo sus imposiciones.

\section{Valor estético}

El valor del cuerpo estético obtiene resultados que lo sitúan en las posiciones más agradables para la muestra de estudiantes en su conjunto, lo cual confirma la perspectiva descrita en la literatura que afirma dicho valor como uno de los más considerados en la sociedad occidental actual. A este respecto, los autores previamente descritos destacan su influencia social en los siguientes términos: Gervilla (2000) sex-ducción, narcisista, estético, Lipovetsky (2000) sexo, narcisismo, estética, García y Alemany (1996, p.106) cuerpo admirable, Ribeiro (2003) apariencia, y Vázquez (2001) belleza.

Según señala Lipovetsky (1994), el narcisismo identifica la apariencia del cuerpo con el ser profundo y liga el problema de la expresión al de la autenticidad. El mundo se preocupa de las arrugas del rostro, de la figura de su silueta en una lucha contra la adversidad temporal queriendo conservar la juventud con cremas antiarrugas y tratamientos para adelgazar. El narcisismo cumple la función de normalización del cuerpo, de obediencia a los imperativos sociales, y es una tendencia dominante en democracias.

\section{Valor intelectual}

El valor del cuerpo intelectual se posicionaba en todas las jerarquías hacia los primeros puestos después del placer mostrando así su agrado hacia dicha dimensión, lo cual coincide con lo relatado a lo largo de la literatura, así por ejemplo el exhaustivo estudio realizado por Gervilla (2000) sobre el concepto de cuerpo a lo largo de la historia.

El ejercicio de la mente, el espíritu o, en su caso, el alma ha sido tradicionalmente contrapuesto al cuerpo, gozando siempre de una mayor estima frente a éste. A este respecto, el dualismo cartesiano del siglo XVI, como queda recogido en el fundamento filosófico del cuerpo, venera la inteligencia, la razón y el pensamiento lógico frente al cuerpo. Recuérdese la famosa frase de Descartes, traducción de Hernández, A. (2005), que resume toda su perspectiva «pienso luego existo», es decir, soy porque pienso, porque tengo un espíritu, no porque tenga un cuerpo que siente, percibe y actúa.

De este modo, el valor intelectual del cuerpo sería uno de los principales valores destacados en la sociedad occidental contemporánea.

\section{Valor afectivo-social}

Los resultados del estudio empírico revelan que el valor afectivo-social es, junto con el biológico, estético e intelectual, uno de los más agradables por detrás del valor placer lo cual puede ser justificado por el fundamento psico-biológico del cuerpo, así como social y filosófico. Cuerpo y afecto son dos realidades indivisibles, es por ello que el valor afectivo-social emerge en la presente investigación como uno de los cinco más valorados o considerados más agradables.

La realidad emocional del cuerpo es inherente a su ser en calidad de humano y está enteramente relacionado con su realidad corporal más material definida por la Biología. En este sentido, Lapierre y Aucouturier (1997) describen la vinculación entre psiquismo y formación neurológica, y Keleman (1997) desarrolla todo un tratado acerca de las relaciones entre Anatomía y emoción bajo el término Anatomía emocional. Keleman (1997, p.15) afirma que «En tanto que proceso 
somático la Anatomía es destino. Es un proceso dinámico vital, un misterio, una incitación, la forma de experiencia que da origen a nuestros sentimientos, pensamientos y acciones». Y prosigue, (Keleman, 1997, p.17), «La vida es formas en movimiento. Somos como secuencias móviles de contornos emocionales variables». Del mismo modo, Schultz (1973, p.10) reafirma la conexión establecida entre estructura y función destacando que «Los puntos corporales muertos indican una falta de integración del individuo en una persona total. Siempre que existe una esfera psicológica que a la persona le resulta difícil asimilar, ya se trate del sexo, la ira, etc, hay un lugar correspondiente en el cuerpo en iguales condiciones».

Sin embargo, pese a la reconocida necesidad e implicación emocional en el cuerpo, la historia siempre ha tratado de esconder cualquier manifestación emocional instintiva. Lapierre y Aucouturier (1980, p.10) destacan que la sociedad occidental rechaza el cuerpo pulsional, premiando la impasibilidad corporal, el dominio de sí mismo, del cuerpo y del lenguaje, legitimando esta conquista como la regla del «saber vivir» y el símbolo de la «buena sociedad», frente a los comportamientos corporales espontáneos, que expresan la afectividad de la emoción y son considerados como «debilidades». La clase burguesa dominante quiere un cuerpo deshumanizado sin debilidades y la conquista del cuerpo es la fuerza.

En este contexto, las implicaciones parecen contrapuestas. Por un lado la naturaleza implica un valor del cuerpo emocional inherente a su ser en calidad de humano, y por otro lado, la sociedad occidental contemporánea define las formas en que esa disposición emocional debe tomar forma, concretamente ocultando su sentir más profundo en beneficio de la norma de linealidad establecida. Este intento de silenciar el cuerpo emocional disfraza sus formas, pero no puede negar su realidad, por lo que el valor afectivo-social del cuerpo es, en todo caso, uno de los principales valores asumidos por el mismo.

\section{Valor ecológico}

El valor ecológico del cuerpo es evidenciado como menos agradable en las tres jerarquías resultantes del estudio empírico, lo cual se ve reafirmado por la revisión bibliográfica llevada a cabo.

La industrialización trajo consigo el éxodo a las ciudades y, con ello, el alejamiento de la Naturaleza. Leyendo a Weil (1995) en sus reflexiones sobre la cultura occidental actual, alejada de la verdadera esencia de la Naturaleza y del contacto con la misma como principal conservadora de la belleza, se puede decir que en la Antigüedad el amor a la belleza del mundo ocupaba un lugar importante en el pensamiento y envolvía la vida entera con una maravillosa poesía. Sin embargo, hoy podría decirse que la raza blanca casi ha perdido la sensibilidad a la belleza del mundo; paradójicamente es casi el único camino por el que podría aparecer la espiritualidad.

Asimismo, es importante destacar la reflexión Cóndor (2008), en sus conversaciones con Albert Hofman, sobre la tecnología como un poderoso factor de desarraigo del hombre sobre la tierra, se basa en la idea de que en la moderna vida urbana todo es artificial, arrancado de las raíces, está muerto, mientras que fuera, al aire libre, se ven los ritmos de la vida de la naturaleza. La urbanización moderna desarraiga al hombre de sus vínculos con la naturaleza.

Con estas citas, se relata el escaso valor ecológico que podría tener el cuerpo en la sociedad occidental actual.

\section{Valores ético y religioso}

Los valores ético y religioso ocupan las últimas posiciones en todas las jerarquías, especialmente el valor religioso que se mantiene siempre en última posición, a excepción de un caso.

Siguiendo a Lypovetsky (1994 y 2000), y retomando sus ideas previamente presentadas, se puede decir que la moral se ha secularizado, se ha independizado de cualquier perspectiva religiosa, pero a la vez, ha caído en manos de nadie, no consagrándose a ningún fin superior. La religión ha sido sustituida por el vacío moral, de tal manera que el ser humano se desvanece ante la falta de ideales, de guías, de referencias, de criterios y de valores. Asimismo, subraya que no hay distinción entre cuerpo y espíritu. El narcisismo se centra en sí mismo y abandona compromisos y militancias religiosas o políticas, ideales y su preocupación axiológica, y se adhiere a la tendencia e inercia actual a falta de reflexión y profundidad más allá de la apariencia.

Como decía Heidegger (2009), no es el tiempo de la filosofía, ni de cualquier aspiración meramente humana.

\section{Valor dinámico}

El valor dinámico es constatado como menos agradable en todas las jerarquías estudiadas, lo cual evidencia la tendencia social actual.

La principal problemática de la sociedad actual puede ser descrita como las tres «s», sedentarismo, sobretrabajo y sobrepeso según Lapresa y Ponde de León (1999). La industrialización trajo consigo la inactividad, el mayor tiempo ocupado a tareas pasivas en el trabajo, y con ello, el sobrepeso. En este sentido, el valor dinámico del cuerpo queda relegado a un segundo plano, no siendo prioritario en la sociedad postmoderna.

\section{Valor instrumental}

El valor instrumental se posiciona entre los valores menos agradables en todas las jerarquías resultantes en el estudio empírico, sin embargo, es destacado por determinados autores como hegemónico en la sociedad occidental contemporánea. Así por ejemplo, García y Alemany (1996, p.106) destacaban el valor instrumental como uno de los cuatro valores más resarcidos en la sociedad actual, si bien, el concepto asociado al valor instrumental relatado por el instrumento de medida utilizado y el asumido por los autores no es enteramente coincidente.

Finalmente, y como síntesis de la comparación de los datos empíricos en estudiantes con los teorizados sobre la sociedad occidental contemporánea, se puede afirmar que los valores del cuerpo resultantes sí son coincidentes en su mayoría.

Contrastando los valores del cuerpo resultantes del estudio empírico con los teorizados a lo largo de la literatura, se observa que los valores del cuerpo placer, biológico, estético, intelectual y afectivo-social son destacados como más 
agradables en el estudio empírico y subrayados como tal en la revisión bibliográfica llevada a cabo. Por el contrario, los valores religioso, ecológico, ético y dinámico son destacados como menos agradables o más desagradables en el estudio empírico y subrayados como tal en la revisión bibliográfica. Por su parte, el valor instrumental es evidenciado como uno de los menos agradables en el estudio empírico, lo cual es contrario a la perspectiva teorizada.

Por ende, queda contrastada la coincidencia entre los valores del cuerpo resultantes del estudio empírico en estudiantes con los teorizados sobre la sociedad occidental contemporánea, puesto que así se produce en nueve de los diez valores del cuerpo.

\section{Conclusiones}

A través de los análisis empíricos efectuados se verifica, con las aportaciones estadísticas pertinentes en cada caso, los supuestos planteados en las consideraciones iniciales, esto es, la confirmación de la existencia de una nueva valorización del cuerpo, un cuerpo fragmentado que lucha por recuperar su esencia natural y humana en un medio hostil que trata de recordar únicamente su realidad material, destacando su reconocimiento como objeto. En el ámbito educativo esta afirmación se torna de especial transcendencia ya que, siguiendo a Águila y López (2019, p.414) «la realidad educativa de hoy mantiene la dualidad mente/cuerpo».

El análisis empírico, realizado sobre una muestra de estudiantes de $1^{\circ}$ de bachillerato en Castilla y León en el curso 2017/18, evidencia cómo los diez valores corporales quedan ordenados jerárquicamente entre los cinco preferidos - placer, biológico, intelectual, estético y afectivo-social-, destacando en todo caso el valor placer como el más valorado; y los cinco menos preferidos - dinámico, ecológico, instrumental, ético y religioso-, siendo el religioso el menos valorado en términos generales. Estos resultados, que son la expresión de la realidad que acontece, coinciden con lo teorizado sobre la sociedad occidental contemporánea. Así, tanto la práctica como la teoría conducen a un nuevo orden axiológico de los valores corporales.

De una parte, la confirmación de esta nueva valorización pone de manifiesto el decisivo peso que los componentes materiales tienen en todas manifestaciones de la sociedad actual. Ello implica, como sugerentemente planteaba Bernard (2006, p.14) «la necesidad de crear y controlar un modo de cultura que nos presente una imagen del cuerpo lo menos alienante posible, que permita la expresión libre de todos los cuerpos en sus deseos y sus acciones recíprocas». La ambición de esta nueva reformulación del cuerpo supondría realmente la reconsideración del mismo, una verdadera reconquista que aúne su realidad más material, su haber intelectual, emocional y existencial-espiritual-moral, es decir, todo un cambio socio-cultural.

Por otro lado, dada la singular relevancia de los valores del cuerpo en los modos de sociabilidad y en la construcción de la identidad personal, el sesgo material actual en la valorización del cuerpo implica la necesidad de revisar los fundamentos teóricos de la educación integral con el propósito de fundamentar la propuesta de pautas de acción educativa y diseño curricular ordenadas a rehabilitar en la cotidianiedad de la escuela, la identidad y valor del cuerpo como estructura y función, superando así cualquier perspectiva dualista. A este respecto, Águila y López (2019, p.417) sostienen que «entender, vivir y estimular el desarrollo de la corporeidad del alumnado puede ayudarle a construir un capital corporal más emancipado y menos sujeto a los modelos estandarizados de la cultura corporal hegemónica».

La educación es continuamente revisada por distintos profesionales y su rumbo es a menudo redirigido en función del presente de la sociedad y el futuro hacia el que quiere evolucionar. Sin embargo, al hablar de educación son pocas las ocasiones en que el cuerpo se torna parte fundamental de tal discusión, cuando es éste el punto de partida y acceso a cualquier realidad. Los valores del cuerpo habrían de ser un objetivo educativo en sí mismo, no pudiendo quedar relegados a lo que definitivamente podría tratarse de currículum oculto.

En este punto emerge la principal prospectiva del presente estudio pretendiendo facilitar intervenciones concretas que enriquezcan el currículum de cara a la formación en valores del cuerpo evitando cualquier fragmentación.

Paralelamente, la literatura especializada acusa importantes diferencias en los valores del cuerpo según diferentes variables de perfil de los estudiantes como pueda ser el sexo o la modalidad de estudios. En el presente estudio se escoge la muestra de manera equilibrada según las cinco variables de perfil con la intención futura de facilitar dicha comparativa. Se ha pretendido definir generalidades que afecten a toda la sociedad, de manera independiente a sus características identificativas. Sin embargo, se plantea como futura línea de investigación la comparación entre las variables de perfil introducidas ya en el presente estudio. Igualmente, se plantea desarrollar un análisis longitudinal que permita estudiar la evolución en el tiempo y un análisis comparativo de diferentes regiones.

\section{Referencias}

Barbero, J. I. (2007). Capital(es) corporal(es) que configuran las corrientes y/o contenidos de la educación física escolar. Agora para la educación física y el deporte, 4, 21 38.

Baudrillard, J. (2002). La ilusión vital. Madrid: Siglo XXI de España Editores, S.A.

Baudrillard, J. (2009). La sociedad de consumo: sus mitos, sus estructuras. Madrid/ : Siglo XXI de España Editores, S.A.

Bernard, M. (2006). El cuerpo. Barcelona: Paidós.

Casares García, P. M., y Collados Zorraquino, J. (1998). Evaluación de valores del cuerpo educando. Revista de ciencias de la educación: Órgano del Instituto Calasanz de Ciencias de la Educación, 174, 237-258.

Cóndor Orduña, M. (trad., Gnoli, A., y Volpi, F. (2008). El Dios de los ácidos: Conversaciones con Albert Hofmann. Madrid/: Ediciones Siruela, S.A.

García, V., y Alemany Briz, C. (1996). El cuerpo vivenciado y analizado. Bilbao: Desclée De Brouwer.

Gervilla Castillo, E. (2000a). Valores del cuerpo educando: antropología del cuerpo y educación. Barcelona/ : Herder. 
Gervilla Castillo, E. (2000b). Un modelo axiológico de educación integral. Revista española de pedagogía, 58 (215), 39-58.

Gervilla Castillo, E. (2002). Valores del cuerpo y educación: ¿qué cuerpo educar? En La presencia del cuerpo en la escuela: XX Congreso Nacional Educación Física y Universidad: Guadalajara, 3-6 de julio de 2002 (pp. 105-126). Alcalá de Henares: Servicio de Publicaciones, Universidad de Alcalá, D.L.

Heidegger, M. (2009). Der Spiegel interview with Martin Heidegger. The Heidegger Reader, transl. J. Veith, 313 333.

Hernández, A. (2005). Descartes: Discurso del método. Alicante, España: Editorial Club Universitario.

Keleman, S. (1997). Anatomía emocional: la estructura de la experiencia somática. Bilbao: Desclée de Brouwer.

Lapierre, A., y Aucouturier, B. (1984). El cuerpo y el inconsciente en educación y terapia. Barcelona/: Editorial Científico-Médica.

Lapierre, A., y Lamarlère, J. (1997). Psicoanálisis y análisis corporal de la relación. Bilbao: Desclée de Brouwer.

Lapresa Ajamil, D., Ponce de León Elizondo, A., y Arana Idiakez, J. (1999). Orientaciones educativas para el desarrollo del deporte escolar. Logroño: Universidad de La Rioja, Mogar Lynotype, S.A.
Le Breton, D. (2002). Signes d'identité: tatouages, piercings et autres marques corporelles. Paris: Editions Métailié. Lipovetsky, G. (2000). Era del vacío. Barcelona: Anagrama.

Lipovetsky, G., Porter, C., y Sennett, R. (1994). The empire of fashion: Dressing modern democracy. Princeton, NJ: Princeton University Press.

Pastor Pradillo, J. L. (2009). El tratamiento de valores en Educación Física. Revista Española de Educación Física y Deportes, 10.

Ribeiro, A. (2003). O corpo que somos: Aparência, sensualidade, comunicação. Lisboa: Editorial Noticias.

Rousseau, J. J. (1996). Discurso sobre el origen de la desigualdad de los hombres. Madrid/ : Alba, D.L.

Schutz, W. (1973). Todos somos uno. La cultura de los encuentros. Buenos Aires: Amorrortu.

Soto, C. Á., \& Vargas, J. J. L. (2019). Cuerpo, corporeidad y educación: una mirada reflexiva desde la Educación Física. Retos: nuevas tendencias en educación física, deporte y recreación, 35, 413-421.

Vázquez Gómez, B. (2001). Los valores corporales y la Educación Física:/ : hacia una reconceptualización de la Educación Física. Agora para la educación física y el deporte, 1,7-17.

Weil, S. (1995). Reflexiones sobre las causas de la libertady de la opresión social. Barcelona-Buenos Aires-México: Paidós.

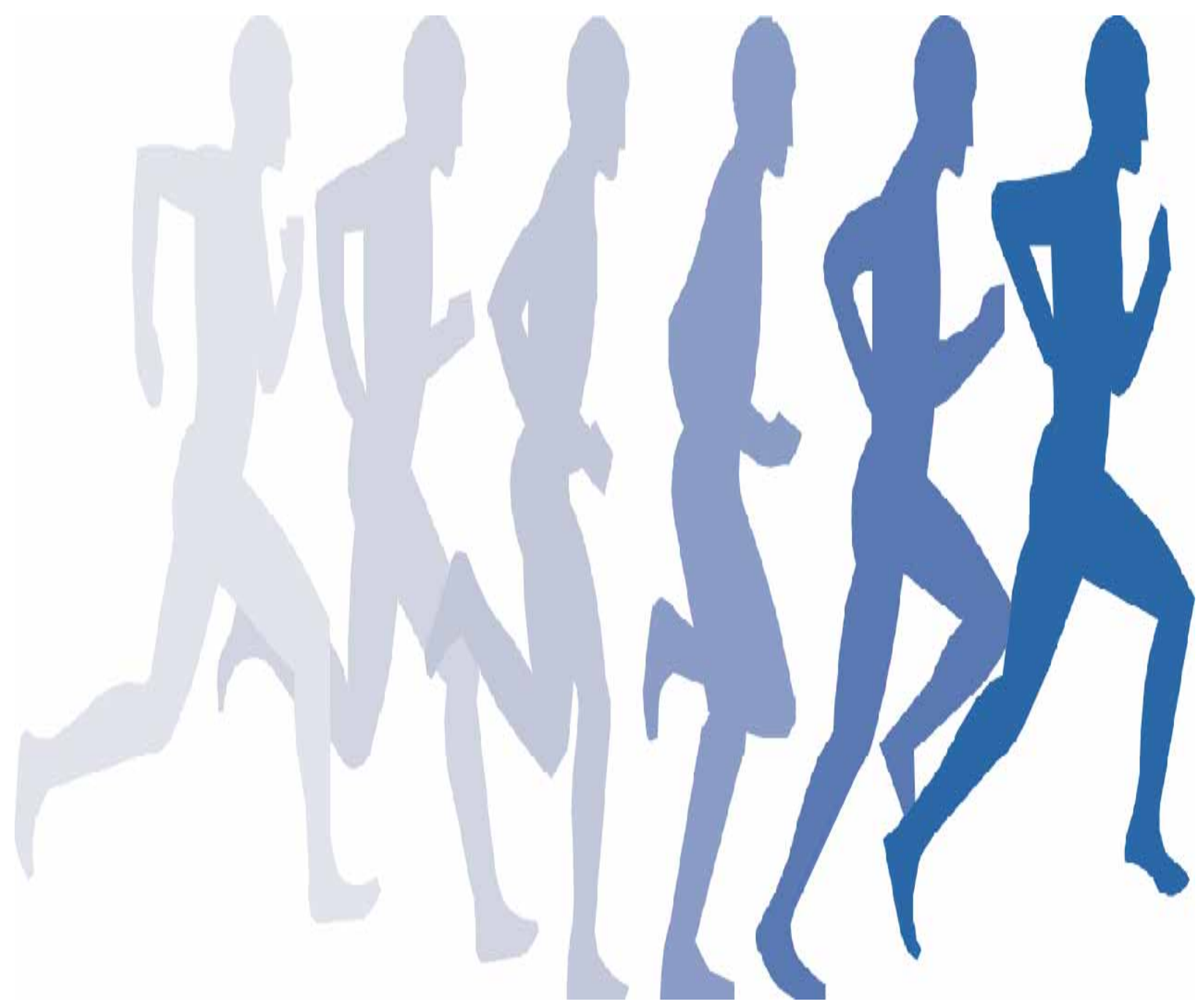

